\title{
A Case of Endometriosis in the Abdominal Wall Post Caesarean Section
}

\author{
Emerson Budhoo, Dale Maharaj* \\ Department of General and Vascular Surgery, St Clair Medical Center, Trinidad and Tobago \\ Email: *dalemaharaj@hotmail.com
}

Received December 18, 2012; revised January 19, 2013; accepted January 28, 2013

\begin{abstract}
We report the case of a 33-year-old female who presented with endometriosis of the anterior abdominal wall following Caesarean Section at the surgical incision site. Abdominal Incisional Site Endometriosis can pose a diagnostic dilema owing to its relative rarity and vagueosity of symptoms, vis-a-vis, cyclical abdominal pain and occasional palpable mass associated with menstruation. A greater index of suspicion should be prompted in such patients especially if symptoms occur following pelvic surgery such as Caesarean Sections, hysterotomy, and myomectomy.
\end{abstract}

Keywords: AISE-Abdominal; Incision; Site; Endometrioma

\section{Introduction}

Endometriosis was first described by Rokitansky in 1860 and is defined as the presence and proliferation of functional endometrial tissue outside the uterine cavity, the commonest site being within the pelvis [1]. Remote from the pelvic area, Extrapelvic endometriosis has been documented in the vulva, urinary tract, gastrointestinal tract, chest wall, lungs and central nervous system [2].

Abdominal wall endometriosis is very uncommon and is usually associated in the scar after previous pelvic surgery [3]. An endometrioma is the development of endometriotic tissue into a distinct mass. We have dubbed this Abdominal Incision Site Endometrioma (AISE).

We present the clinical findings, radiologic features and treatment of a patient with AISE and propose that the condition is not as rare as once thought due to misdiagnosis.

\section{Case Report}

A 33-year-old female presented with a one year history of intermittent abdominal pain in her suprapubic region. The pain became worse during her menses and has increased progressively with time. For the past year she has been complaining of a vague swelling in her abdomen that was increasing in size.

The patient had her menarche at the age of 13 years. Thereafter she had 27-day to 28-day menstrual cycles and menstrual periods lasting 6 to 7 days with normal blood loss. She had a delivery two years ago via Caesar-

*Corresponding author. ean Section.

Examination findings revealed an ill-defined mass in the suprapubic region, $1 \mathrm{~cm}$ left of the midline measuring, $1.5 \mathrm{~cm}$ by $1.5 \mathrm{~cm}$ which was extremely tender and firm. The tenderness persisted with contraction of her rectus abdominis muscles, implying extraperitoneal pathology (Carnett's Sign). A healed Pfannenstiel incision was noted just inferior to the mass. No inguinal lymph nodes were palpated. All other examination findings were essentially normal.

The diagnosis of AISE was entertained and an MRI of her abdomen including her anterior abdominal was performed to determine the extent of the lesion. A $2 \mathrm{~cm}$ by $1.5 \mathrm{~cm}$ mass with increased vascularity was noted. The mass was confined to the subcutaneous tissue and superior to the rectus muscle (Figure 1).

The mass was completely excised along with surrounding tissues and an abdominoplasty was performed. The cut specimen demonstrated a fatty mass with collections of clotted blood. Histology revealed endometrial glandular tissue with clear excision margins.

\section{Discussion}

Endometriosis is defined as the presence of functional endometriotic tissue outside the uterine cavity [4-6]. Endometriosis most commonly affects the pelvic organs, with the ovary being affected in $30 \%-40 \%$ of cases and followed by the uterine peritoneum, the fallopian tubes, the bladder, the rectum, the vulva, and the pelvic ligaments in decreasing order of frequency.

Extrapelvic endometriosis has been documented in the 


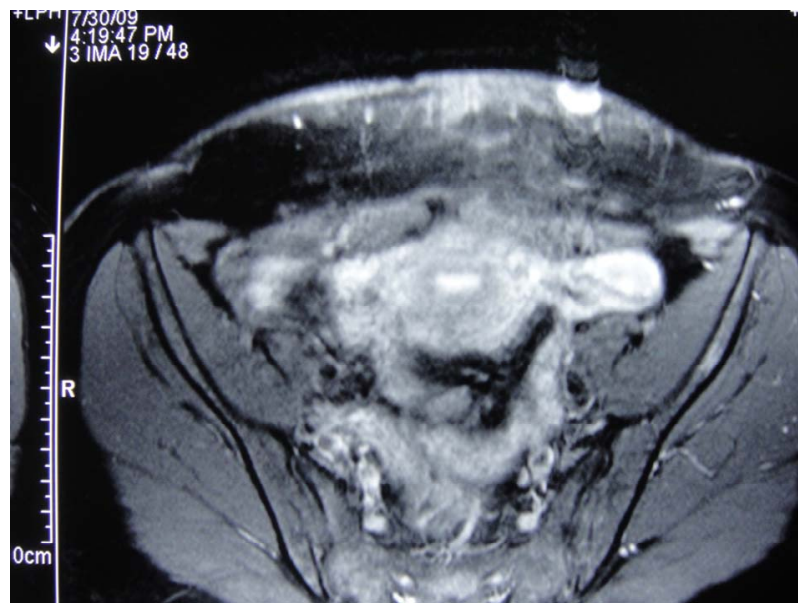

Figure 1. MRI of abdomen axial cut showing endometrioma superficial to rectus abdominis.

bladder, kidney, bowel, lymph nodes, lungs, umbilicus, hernia sacs, and abdominal wall [7]. Abdominal wall endometriosis usually involves incision sites from hysterectomy, laparoscopic port sites and Cesarean section, which accounts for $0.03 \%$ to $0.4 \%$ of endometriosis [8]. The endometriotic deposit is usually confined to the abdominal skin and subcutaneous tissue with sparing of the muscle and rectus sheath. In fact, endometriosis involving the rectus abdominis muscle is very rare. To date, only 19 such cases have been reported in the medical literature [9] since it was first described in 1984 by Amato and Levitt [10].

Like other forms of endometriosis, AISE is associated with periodic pain, odor, itch and bleeding corresponding to the menstrual cycle [3]. There may be an associated mass that can become tense and tender due to recurrent bleeding and fibrotic scar formation. Carnette's test may be useful in localizing the pathology to an extraperitoneal or more superficial subcutaneous site and may be useful when MRI scanning is not readily available.

Several diagnostic tools can be used to confirm the diagnosis. Ultrasonography and computed tomography may demonstrate and confirm the superficial nature of the mass. The gold standard is magnetic resonance imaging which can detect small lesions and confirm its vascularity as it did in this case.

Fine needle aspiration cytology FNAC can confirm the diagnosis of AISE by demonstrating epithelial cells, stromal cells and hemosiderin laden macrophages. FNAC can however seed the needle tract and spread endometrial tissue [11].

Cancer antigen 125 (CA-125) is elevated in endometriosis and can be used to monitor treatment. It however is also elevated in gynecologic malignancies and is therefore not diagnostic [12].

The development of the mass usually occurs 1 - 2 years after the pelvic surgery [13]. Some of the differentials for
AISE include abscess formation, hematoma, suture granuloma, inguinal hernia, sarcoma, and metastatic cancer. However these conditions can easily ruled out with a proper clinical evaluation and imaging.

Medical management using oral contraceptive, danazol, gonadotropin-releasing hormone agonists, progesterones, aromatase inhibitors, and transcutaneous nerve stimulation have all been employed [14,15]. These modalities can produce decidualization, atrophy of endometrial tissue, and for relieving pain, but recurrence after cessation of medication and the untoward side effects makes surgical resection the treatment of choice $[3,6$, $7,16]$.

Standard surgical management involves the excision of all endometriotic tissue including skin, subcutaneous tissue and rectus fascia with muscle when involved. Excision of the anterior abdominal wall musculature may require mesh reconstruction [17]. In our case, complete excision of the AISE tissue was performed down to the fascial tissue. To compensate for the potentially large asymmetric and aesthetically displeasing wound closure, we performed a mini-abdominoplasty, removing the excess, striated lower quadrant postpartum skin.

In conclusion, AISE is not a common finding, but we believe that it is an underdiagnosed clinical entity. At the risk of sounding cliché, a detailed history, as well as a thorough examination will likely reveal the diagnosis and can be further supported by appropriate imaging techniques (ultrasound, CT or MRI). Whenever the diagnosis is uncertain, FNAC can be used to obtain a cellular diagnosis. Total wide excision of the lesion is the treatment of choice and can be safely combined with a "minitummy-tuck" to produce a cosmetic scar.

\section{REFERENCES}

[1] C. Von Rokitansky, "Ueber Uterusdrüsen-Neubildung in Uterus- und Ovarial-Sarcomen,” Ztsch K K Gesellsch der Aerzte zu Wien, Vol. 37, 1860, pp. 577-581.

[2] G. M. Honore, "Extrapelvic Endometriosis,” Clinical Obstetrics and Gynecology, Vol. 42, 1999, p. 711.

[3] S. Gourgiotis, G. Veloudis, N. Pallas, P. Lagos, N. S. Salemis and C. Villias, "Abdominal Wall Endometriosis: Report of Two Cases,” Romanian Journal of Morphology and Embryology, Vol. 49, No. 4, 2008, pp. 553-555.

[4] P. J. Woodward, R. Sohaey and T. P. Mezzetti, "Endometriosis: Radiologic-Pathologic Correlation,” Radiographics, Vol. 21, No. 1, 2001, pp. 193-216.

[5] D. L. Olive and L. B. Schwartz, "Endometriosis,” New England Journal of Medicine, Vol. 328, 1993, pp. 17591769. doi:10.1056/NEJM199306173282407

[6] T. M. Yi, Y. J. Kim, S. G. Kim, et al., "A Case of Endometrioma at the Abdominal Scar," Journal of the Korean Society of Plastic and Reconstructive Surgeons, Vol. 37, 2010, pp. 843-846. 
[7] K. B. Gajjar, A. A. Mahendru and M. A. Khaled, "Caesarean Scar Endometriosis Presenting as an Acute Abdomen: A Case Report and Review of Literature,” Archives of Gynecology and Obstetrics, Vol. 277, No. 2, 2008, pp. 167-169. doi:10.1007/s00404-007-0431-4

[8] E. T. Lee, H. M. Park, D. G. Lee, K. J. Shin, H. S. Kim, R. H. Sung and D. H. Ryu, "Application of Mini-Abdominoplasty after Conservative Excision of Extensive Cesarean Scar Endometriosis,” Archives of Plastic Surgery, Vol. 39, No. 5, 2012, pp. 551-555. doi:10.5999/aps.2012.39.5.551

[9] P. G. Calò, R. Ambu, F. Medas, A. Longheu, G. Pisano and A. Nicolosi, "Rectus Abdominis Muscle Endometriosis Report of Two Cases and Review of the Literature," Annali Italiani di Chirurgia, 2012.

[10] M. Amato and R. Levitt, "Abdominal Wall Endometrioma: CT Findings," Journal of Computer Assisted Tomography, Vol. 8, No. 6, 1984, pp. 1213-1214. doi:10.1097/00004728-198412000-00040

[11] P. Veda and M. Srinivasaiah, "Incisional Endometriosis: Diagnosed by Fine Needle Aspiration Cytology,” Journal of Laboratory Physicians, Vol. 2, No. 2, 2010, pp. 117120. doi:10.4103/0974-2727.72216

[12] M. Szubert, J. Suzin, T. Wierzbowski and K. Kowalczyk-
Amico, “CA-125 Concentration in Serum and Peritoneal Fluid in Patients with Endometriosis-Preliminary Results,” Archives of Medical Science, Vol. 8, No. 3, 2012, pp. 504-508. doi:10.5114/aoms.2012.29529

[13] P. Goel, S. S. Sood, A. Dalal and Romilla, "Cesarean Scar Endometriosis-Report of Two Cases," Indian Journal of Medical Sciences, Vol. 59, No. 11, 2005, pp. 495498. doi:10.4103/0019-5359.18967

[14] I. E. Sasson and H. S. Taylor, “Aromatase Inhibitor for Treatment of a Recurrent Abdominal Wall Endometrioma in a Postmenopausal Woman," Fertility and Sterility, Vol. 92, No. 3, 2009, p. 1170. doi:10.1016/j.fertnstert.2009.05.071

[15] A. Prentice, “Endometriosis: Clinical Review,” British Medical Journal, Vol. 32, 2010, pp. 93-95.

[16] L. Giannella, A. La Marca, G. Ternelli and G. Menozzi, "Rectus Abdominis Muscle Endometriosis: Case Report and Review of the Literature," Journal of Obstetrics and Gynaecology Research, Vol. 36, No. 4, 2010, pp. 902-906. doi:10.1111/j.1447-0756.2010.01236.x

[17] C. Nissotakis, E. Zouros, K. Revelos and G. H. Sakorafas, "Abdominal Wall Endometrioma: A Case Report and Review of the Literature,” AORN Journal, Vol. 91, No. 6, 2010, pp. 730-742. doi:10.1016/j.aorn.2010.01.014 